\title{
Investigating the Performance of Apodized Fiber Bragg Gratings for Sensing Applications
}

\author{
I. Ashry ${ }^{1,4}$, A. Elrashidi ${ }^{2,4}$, A. Mahros ${ }^{3,4}$, M. Alhaddad, and K. Elleithy
}

\begin{abstract}
Fiber Bragg gratings (FBGs) technology has demonstrated its suitability for many applications in recent fiber technologies. Sensing application is one of the main applications of FBGs. In this work, we present a comprehensive investigation for using apodized FBGs in sensing applications. Different evaluation parameters such as, reflectivity, sidelobes, and fullwidth-half-maximum (FWHM) are tested in order to determine the most proper apodization profile for sensors. According to our study, the Blackman apodization gives the best profile that can be used in sensing applications. The reflectivity of Blackman apodization is nearly unity with minimum sidelobes level, $-\mathbf{- 6 0 . 3}$ dB, and narrow FWHM. The length of Blackman apodized FBG is $0.33 \mathrm{~cm}$ and $\Delta n=14.4 \times 10^{-4}$ and maximum reflectivity is $99.44 \%$.
\end{abstract}

Index Terms-Fiber Bragg grating, Apodization, Sensors, DWDM, and FWHM.

\section{INTRODUCTION}

A major drawback to the evolution of optical fiber-based networks has been its reliance on bulk optics for conditioning and controlling the guided light beam. The necessity of coupling light out of the waveguide to perform, for example, reflection, diffraction, amplification, and filtering is an inherently lossy process [1]. Moreover, coupling light in and out of fiber requires stringent tolerance on optical alignment which makes a simple system to be complicated and expensive in practice. Replacing a bulk optic mirror or beam

Manuscript received February 5, 2014.

${ }^{1}$ I. Ashry is with the Department of Electronics and Communications Engineering, College of Engineering and Information Technology, University of Business and Technology, Jeddah 21432, Saudi Arabia (e-mail: i.ashry@ubt.edu.sa).

${ }^{2}$ A. Elrashidi is with the Department of Electronics and Communications Engineering, College of Engineering and Information Technology, University of Business and Technology, Jeddah 21432, Saudi Arabia (phone: +966-561127894; e-mail: a.elrashidi@ubt.edu.sa).

${ }^{3}$ A. Mahros is with the Department of Physics, King Abdulaziz University, Jeddah 21432, Saudi Arabia (amr.mahros@mena.vt.edu)

${ }^{4}$ Department of Engineering Mathematics and Physics, Alexandria University, Alexandria 21544, Egypt.

M. Alhaddad is with the Department of Electrical Engineering, College of Engineering and Information Technology, University of Business and Technology, Jeddah 21432, Saudi Arabia (e-mail: mmhaddad@ cba.edu.sa).

K. Elleithy is with the Department of Computer and Electrical Engineering, Faculty of Engineering, University of Bridgeport, CT 06604, USA (e-mail: elleithy@bridgeport.edu). splitter with a fiber equivalent can increase the system stability and reduce the overall size.

With the significant discovery of photosensitivity in optical fibers, a new class of infiber component has been developed, called the fiber Bragg grating (FBG) [2,3]. This device can perform many of the aforementioned primary functions, such as reflection and filtering in a highly efficient and low loss manner. FBG is a comparatively simple device and its most basic form consists of a periodic modulation of the index along the fiber core.

The main peak in the reflection spectrum of a finite length FBG with uniform modulation of the index of refraction is accompanied by a series of sidelobes at adjacent wavelengths. It is important in some applications to lower and, if possible, to eliminate the reflectivity of these sidelobes, or to apodize the reflection spectrum of the grating. For example, in dense wavelength division multiplexing (DWDM) it is important to have very high rejection of the nonresonant light in order to eliminate cross talks between information channels [4], and therefore, apodization becomes absolutely necessary.

FBGs have been used in a myriad of applications such as, lasers [5,6], optical delay buffers [7], filters [8], and multiplexers/demultiplexers [9]. Furthermore, FBGs are extensively used for sensing applications [10]. In particular, FBG can sense strain and temperature with high accuracy conditioning that, it has high reflectivity, low sidelobes, and narrow full-width-half-maximum (FWHM).

Though apodization is a well known technique used in many FBG applications, few literature reviews deal with optimizing sensors performance using apodized FBG [11-13]. Also, earlier literature studies lack demonstrating the effect of large variety of apodization profiles on the performance of FBGbased sensors.

In this paper, we introduce a comprehensive study on the impact of using different apodization profiles (Uniform, Sine, Sinc, Gaussian, Hamming, Tanh, Bartlett, Blackman, Cauchy, and Raised sine) on the performance of FBG-based sensors. The performance evaluation parameters tested in this study are FBG reflectivity, sidelobes level, and FWHM.

\section{II.BACKGROUND AND THEORY}

FBG filter is defined as a periodic perturbation of the refractive index along the fiber axial, which is formed by exposing the fiber core to an intense ultraviolet (UV) optical 
interference pattern. FBG fabrication was first demonstrated by Hill et al. [3]. The authors experimentally demonstrated that the UV light at $248 \mathrm{~nm}$ could be used to form gratings that would reflect any wavelength by illuminating the fiber through the side of the cladding with two intersecting beams of UV laser lights. Reflectivity approaching $100 \%$ is possible, with the grating FWHM tailored from typically $0.1 \mathrm{~nm}$ to in excess of $100 \mathrm{~nm}$ [3].

Consider a uniform FBG formed within the core of an optical fiber with an average refractive index $n_{\mathrm{o}}$. The refractive index profile, shown in Figure 1, can be expressed as [3]:

$n=n_{o}+\Delta n(z) \cos \left(\frac{2 \pi}{\Lambda} z\right)$,

where $\Delta n$ represents the amplitude of the induced refractive index perturbation, $z$ is the distance along the FBG longitudinal axis, and $\Lambda$ denotes the grating spacing. The Bragg wavelength, $\lambda_{\mathrm{B}}$, is related to the effective grating index $n_{\text {eff }}$ as:

$\lambda_{\mathrm{B}}=2 n_{\text {eff }} \Lambda$.

Using the coupled-mode theory, the reflectivity of the FBG is given by [3]:

$R(L, \lambda)=\frac{k^{2} \sinh ^{2}(s L)}{s^{2} \cosh ^{2}(s L)+\delta^{2} \sinh ^{2}(s L)}$,

where $R(L, \lambda)$ represents the reflectivity function of the grating length $L$ and wavelength $\lambda, k$ denotes the absolute value of the coupling coefficient, $\delta$ represents the detuning from the Bragg wavelength, and $s^{2}=k^{2}-\delta^{2}$. The absolute value of the coupling coefficient, $k$, for sinusoidal variation of index perturbation along the fiber axis is given by:

$k(z)=\frac{\eta \pi \Delta n(z)}{\lambda_{B}}$,

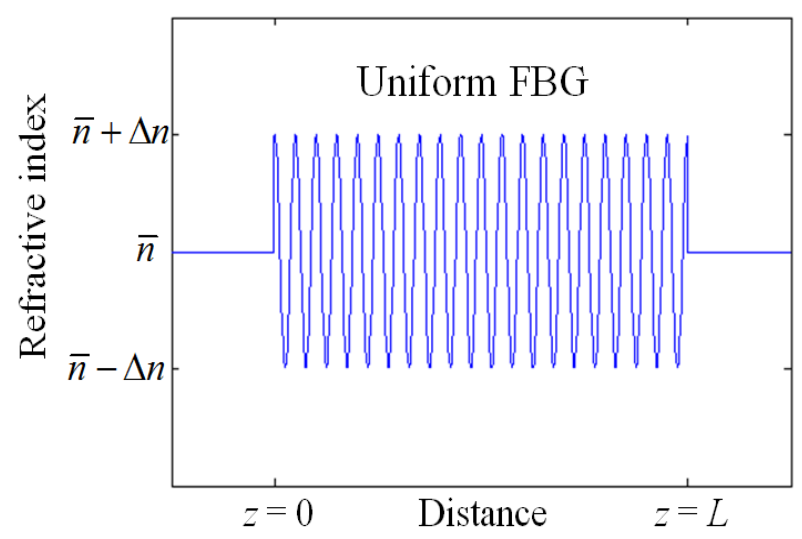

Fig. 1. Refractive index change along uniform FBG.

where $\eta$ is defined as the fraction of the fiber mode power contained within the fiber core. The sidelobes of the FBG may be removed by apodizing the grating. Apodization is a process of tapering the strength of the grating at either end so that the apparent refractive index change is gradual rather than abrupt such that $\Delta n(z)$, Eq. (1), is a slowly varying function with $z$, as shown in Figure 2, and the reflectivity in this case can be calculated using the transfer matrix method [3].

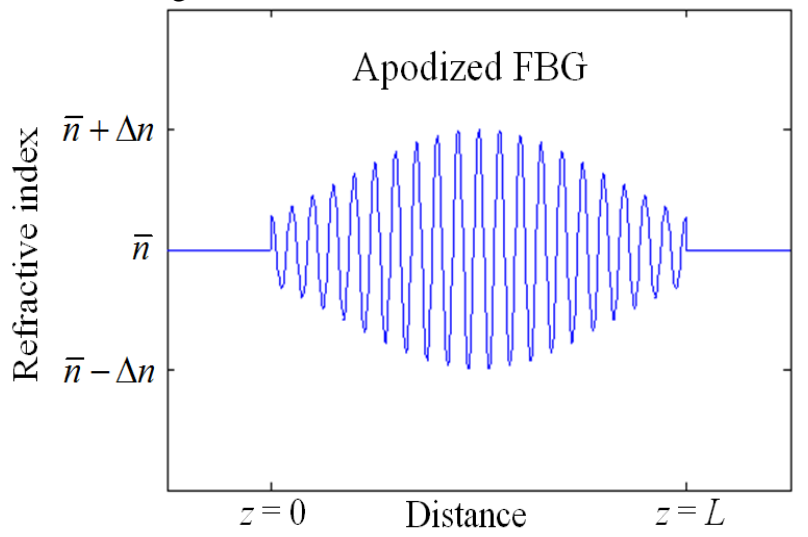

Fig. 2. Refractive index change along apodized FBG.

The main apodization profiles considered in the present work are [6]:

1. sine:

$\Delta n(z)=\Delta n\left[\sin \left(\frac{\pi z}{L}\right)\right], \quad 0 \leq z \leq L$

2. sinc:

$\Delta n(z)=\Delta n\left[\frac{\sin (x)}{x}\right], \quad x=\frac{2 \pi\left(z-\frac{L}{2}\right)}{L}, \quad 0 \leq z \leq L$

3. Gaussian:

$\Delta n(z)=\Delta n\left[-5\left(\frac{\left(z-\frac{L}{2}\right)}{L}\right)^{2}\right], \quad 0 \leq z \leq L$

4. Hamming:

$\Delta n(z)=\Delta n\left[\frac{1+H \cos \left(\frac{2 \pi\left(z-\frac{L}{2}\right)}{L}\right)}{1+H}\right], H=0.9,0 \leq z \leq L$

\section{Tanh:}

$$
\begin{aligned}
\Delta n(z) & =\Delta n\left[\tanh \left(\frac{8 z}{L}\right)\right], & & 0 \leq z \leq \frac{L}{2} \\
& =\Delta n\left[\tanh \left(\frac{8(L-z)}{L}\right)\right], & & \frac{L}{2} \leq z \leq L
\end{aligned}
$$

\section{Bartlett:}

$$
\begin{aligned}
\Delta n(z) & =\Delta n\left[\frac{2 z}{L}\right], & & \leq z \leq \frac{L}{2} \\
& =\Delta n\left[-2\left(\frac{z}{L}-1\right)\right], & & \frac{L}{2} \leq z \leq L
\end{aligned}
$$

\section{Blackman:}




$$
\begin{aligned}
& \Delta n(z)=\Delta n\left[\frac{1+1.19 \cos (x)+0.19 \cos (2 x)}{2.38}\right] \\
& x=\frac{2 \pi\left(z-\frac{L}{2}\right)}{L}, \quad 0 \leq z \leq L
\end{aligned}
$$

\section{Cauchy:}

$\Delta n(z)=\Delta n\left[\frac{1-\left(\frac{2\left(z-\frac{L}{2}\right)}{L}\right)^{2}}{1-\left(\frac{2 C\left(z-\frac{L}{2}\right)}{L}\right)^{2}}, \quad C=0.5, \quad 0 \leq z \leq L\right.$

\section{Raised sine:}

$\Delta n(z)=\Delta n\left[\sin ^{2}\left(\frac{\pi z}{L}\right)\right], \quad 0 \leq z \leq L$

The various apodization profiles are plotted in Figure 3.

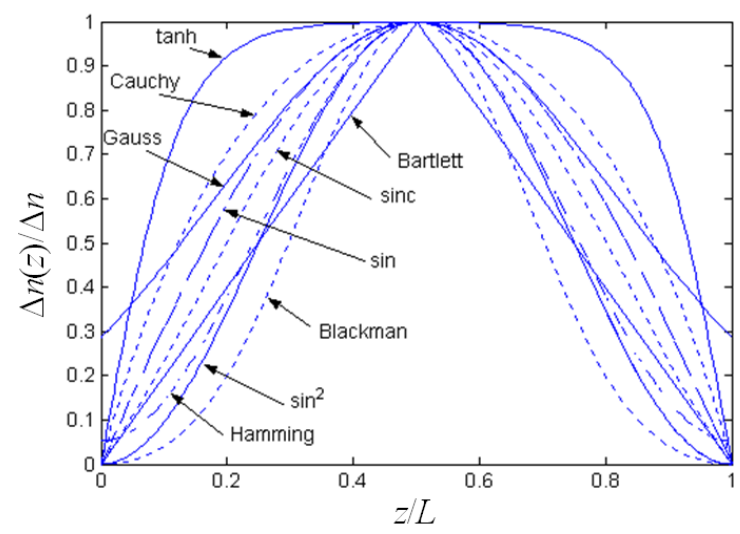

Fig. 3. Apodization profiles.

\section{RESULTS AND DISCUSSIONS}

The performance of the different apodization profiles are tested based on investigating three different parameters; maximum reflectivity, sidelobes level, and FWHM. In this work, the simulations are performed for a single mode step index fiber of $1550 \mathrm{~nm}$ Bragg wavelength. The length of the used FBG is within the range of $0.1 \mathrm{~cm}$ to $1.9 \mathrm{~cm}$ and $\Delta n$ is selected to be between $0.01 \times 10^{-4}$ (weak grating) and $14.4 \times 10^{-4}$ (strong grating). These values are selected to meet previous literature reviews [11-13].

\section{A. Maximum reflectivity}

The maximum reflectivity of different apodization profiles is compared using constant $L$ and $\Delta n$. In this section, we use $L$ $=1 \mathrm{~cm}$ and $\Delta n=2 \times 10^{-4}$ which provides maximum reflectivity of near unity for the uniform FBG. Using the transfer matrix method, we can get the reflectivity of each apodized FBG. It is observed that, apodization reduces the maximum reflectivity with respect to that of uniform FBG and this appears on the normal scale of reflectivity (Figure 4). The maximum reflectivity of each apodization profile is summarized in Table 1 .

TABLE 1

MAXIMUM REFLECTIVITY OF APODIZED FBGS

\begin{tabular}{|c|c|}
\hline Apodization profile & Maximum reflectivity \\
\hline Uniform & $99.44 \%$ \\
\hline sine & $94.10 \%$ \\
\hline sinc & $92.04 \%$ \\
\hline Gaussian & $96.13 \%$ \\
\hline Hamming & $88.19 \%$ \\
\hline tanh & $98.27 \%$ \\
\hline Bartlett & $86.12 \%$ \\
\hline Blackman & $77.66 \%$ \\
\hline Cauchy & $96.17 \%$ \\
\hline Raised sine & $\% 86.12$ \\
\hline
\end{tabular}
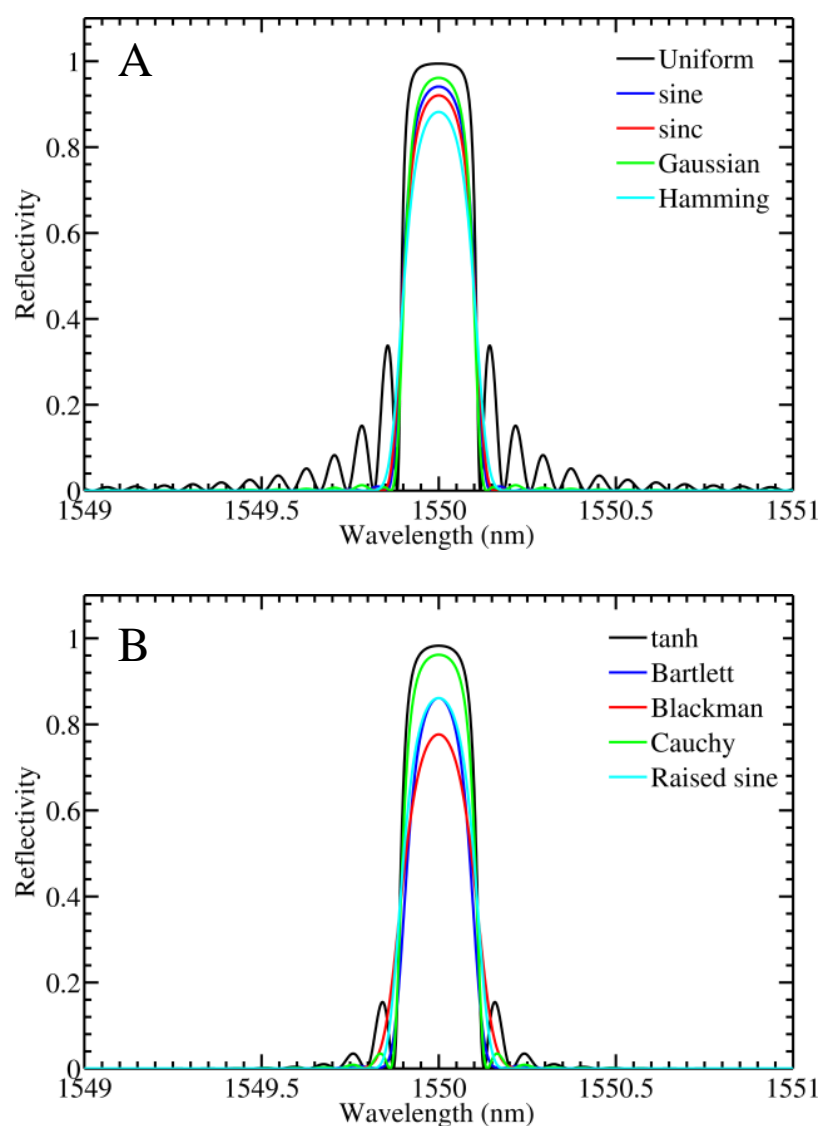

Fig. 4. Reflectivity versus wavelength of different apodized FBGs at $L=1 \mathrm{~cm}$ and $\Delta n=2 \times 10^{-4}$.

The results in Table 1 show that, the Blackman apodization profile has the minimum value of maximum reflectivity among the remaining apodization profiles. 


\section{B. Sidelobes level}

The sidelobes level of different apodization profiles are compared using constant $L$ and $\Delta n$ that are used in the previous section. The apodization has a significant impact on reducing the sidelobes found in uniform FBG. The sidelobes level reductions caused by different apodization profile are illustrated in Figure 5, when the reflectivity is represented on logarithmic scale. Table 2 summarizes the values of the main sidelobe level of each apodization profile.

TABLE 2

MAIN SIDELOBE LEVEL OF APODIZED FBGs

\begin{tabular}{|c|c|}
\hline Apodization profile & $\begin{array}{c}\text { Main sidelobe level } \\
(\mathrm{dB})\end{array}$ \\
\hline Uniform & -4.7 \\
\hline sine & -20.1 \\
\hline sinc & -25.9 \\
\hline Gaussian & -21.0 \\
\hline Hamming & -47.0 \\
\hline tanh & -8.1 \\
\hline Bartlett & -21.9 \\
\hline Blackman & -60.3 \\
\hline Cauchy & -14.6 \\
\hline Raised sine & -34.6 \\
\hline
\end{tabular}
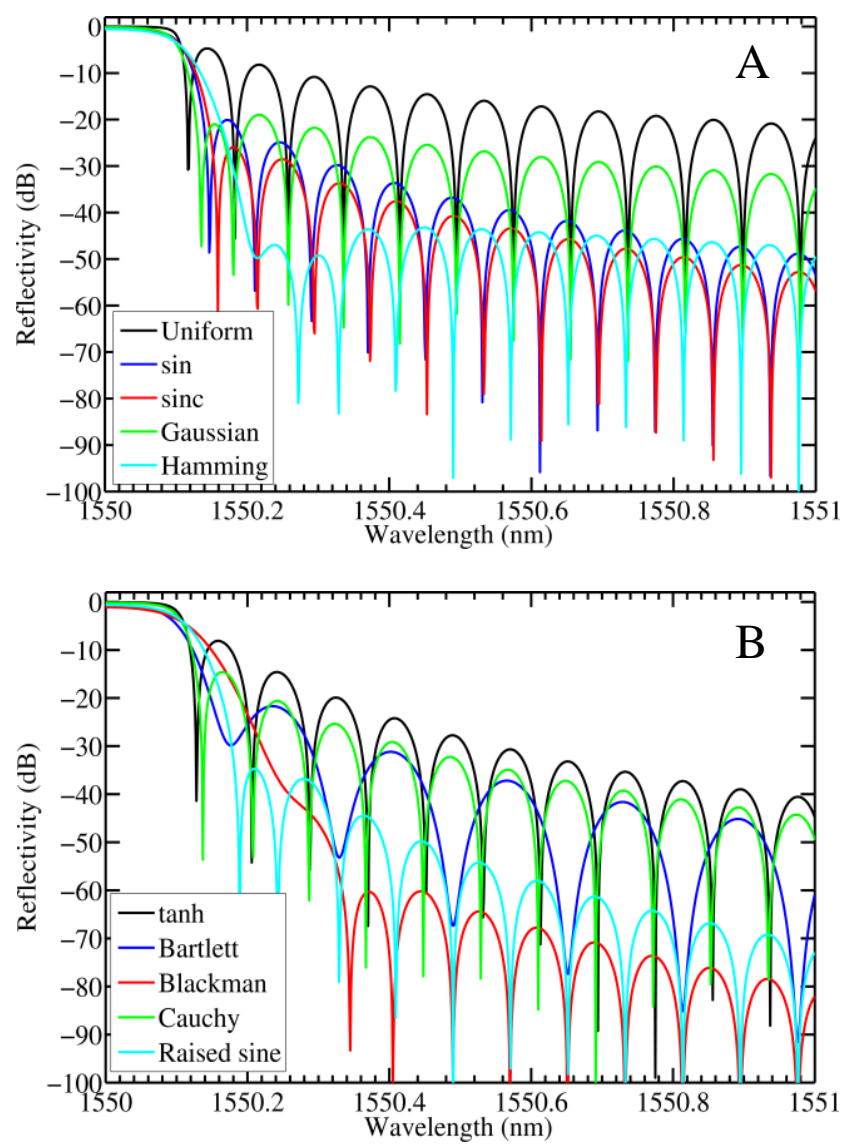

Fig. 5. Reflectivity in $\mathrm{dB}$ versus wavelength of different apodized FBGs at $L=1 \mathrm{~cm}$ and $\Delta n=2 \times 10^{-4}$.

The results in Table 2 show that, the Blackman profile is the best apodization method for reducing the sidelobes level.

\section{Full-width-half-maximum}

As mentioned previously, FBGs used for sensing applications should have high reflectivity, low sidelobes level, and narrow FWHM. The obtained results in the previous two sections show that, Blackman apodization profile has minimum level of sidelobes. However, its maximum reflectivity is the worst. As a result, we aim to increase the maximum reflectivity of the Blackman apodized FBG such that it becomes similar to the uniform FBG. Simultaneously, narrow FWHM is another target.

The maximum reflectivity value is found to be dependent on the fiber length and $\Delta n$. Figure 6 and Figure 7 show the change of the maximum reflectivity with $L$ and $\Delta n$, simultaneously. As a result, in order to increase the maximum reflectivity of the Blackman apodized FBG, we can either increase the FBG length and/or $\Delta n$.

We first increase the length of the Blackman apodized FBG to be $1.9 \mathrm{~cm}$, maximum value used in the literature, and changes $\Delta n$ till the maximum reflectivity becomes $99.44 \%$ (maximum reflectivity of uniform FBG). Figure 8 (dashed blue line) illustrates the reflectivity of Blackman apodized FBG at $L=1.9 \mathrm{~cm}$ and $\Delta n=2.5 \times 10^{-4}$ in which the maximum reflectivity is $99.44 \%$.

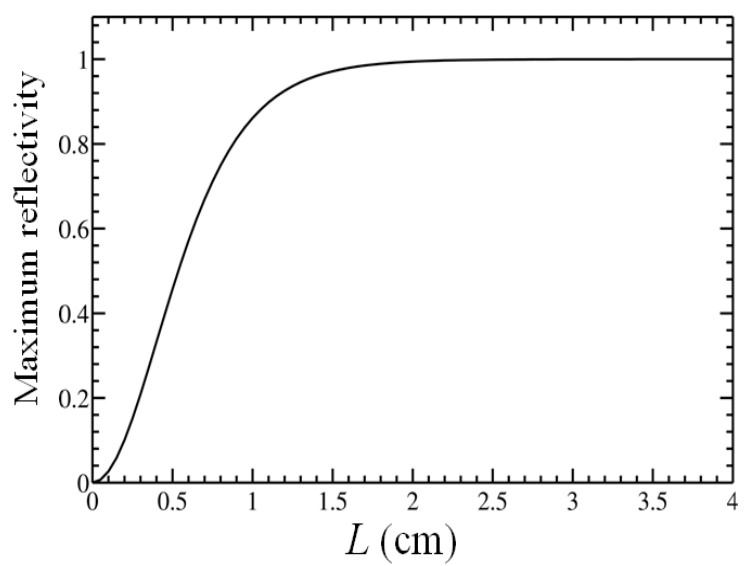

Fig. 6. Change of maximum reflectivity with $L$.

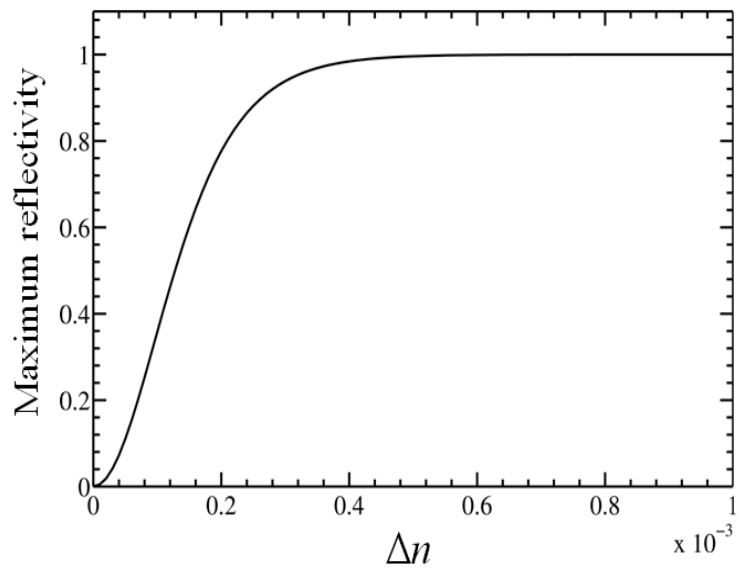

Fig. 7. Change of maximum reflectivity with $\Delta n$. 

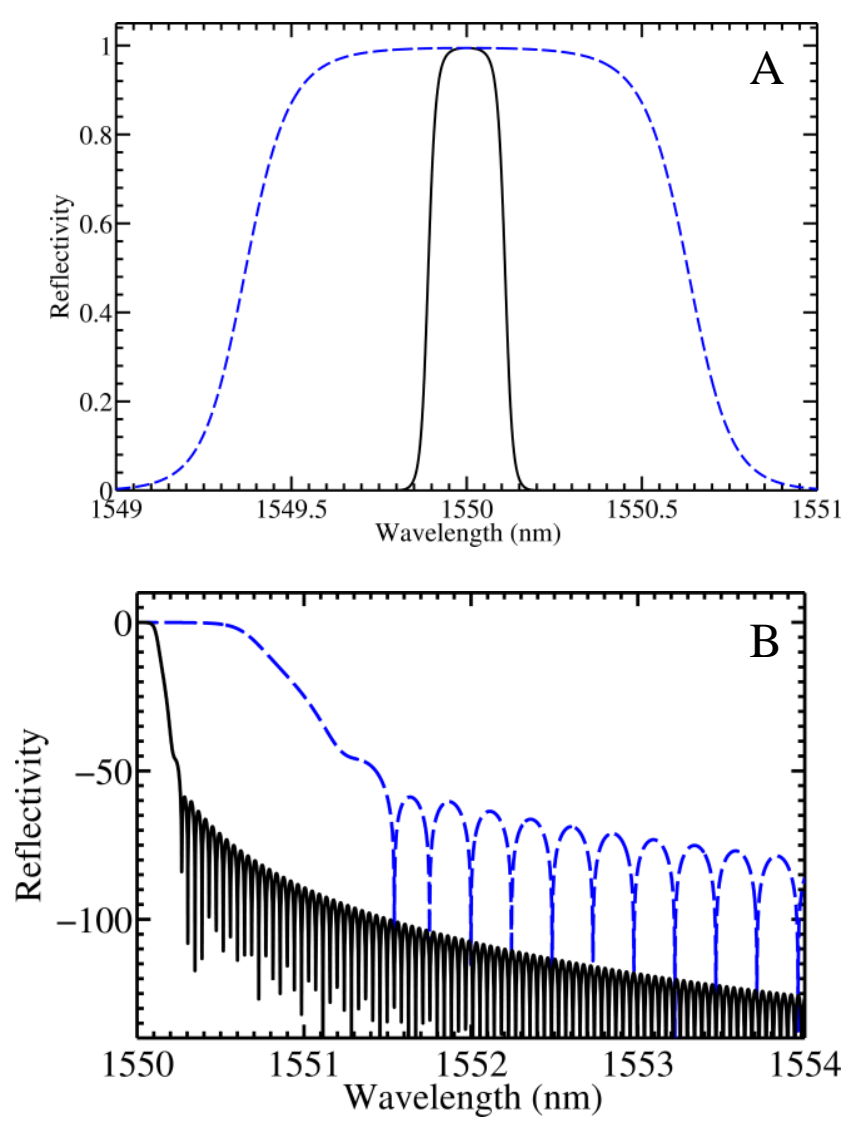

Fig. 8. Reflectivity of Blackman apodized FBG at $L=1.9 \mathrm{~cm}$ and $\Delta n=2.5 \times 10^{-4}$ (dashed blue line) and at $L=0.33 \mathrm{~cm}$ and $\Delta n=14.4 \times 10^{-4}$ (sold black line) on (A) normal and (B) logarithmic scale.

Similarly, we then increase $\Delta n$ to be $14.4 \times 10^{-4}$, maximum value used in the literature, and we change $L$ till the maximum reflectivity becomes $99.44 \%$ (maximum reflectivity of uniform FBG). Figure 8 (solid black line) illustrates the reflectivity of Blackman apodized FBG at $L=0.33 \mathrm{~cm}$ and $\Delta n=14.4 \times 10^{-4}$ in which the maximum reflectivity is $99.44 \%$.

The results obtained in Figure 8 show that increasing the FBG length is better than increasing $\Delta n$. This because the longer FBG has narrower FWHM and low average sidelobes level. As a result, we conclude that, Blackman apodized FBG is the best apodization profile that can be used in sensing applications conditioning that its maximum reflectivity increases by increasing its length.

\section{CONCLUSION}

In this paper, we present a comprehensive investigation of the performance of different apodization profiles that are used for sensing applications. The evaluation parameters used for comparing the apodization profiles are FBG reflectivity, sidelobes level, and FWHM. Blackman apodization is found to be the best profile to be used for sensing applications because its reflectivity can nearly reach unity with minimum sidelobes level and narrow FWHM. Blackman apodized FBG of $0.33 \mathrm{~cm}$ length and $\Delta n=14.4 \times 10^{-4}$ can have maximum reflectivity of $99.44 \%$.

\section{REFERENCES}

[1] J. T. Kim, S-W. Jung, S-H Ahn, C-G Choi, and M-U Jeong, "A reflective curved mirror with low coupling loss for optical interconnection," IEEE Phot. Technol. Lett., vol. 16, pp. 185-187, Jan. 2004.

[2] O. I. Medvedkov, S. A. Vasiliev, P. I. Gnusin, and E. M. Dianov, "Photosensitivity of optical fibers with extremely high germanium concentration," Opt. Mat. Express, vol. 2, pp. 1478-1489, Oct. 2012.

[3] A. Othonos, and K. Kalli, Fiber Bragg Gratings: Fundamentals and Applications in Telecommunications and Sensing. London: Artech House, 1999, ch. 5.

[4] R. J. Williams, C. Voigtländer, G. D. Marshall, A. Tünnermann, S. Nolte, M. J. Steel, and M. J. Withford, "Point-by-point inscription of apodized fiber Bragg gratings," Opt. Lett., vol. 36, pp. 2988-2990, Aug. 2011.

[5] J-B. Lecourt, C. Duterte, F. Narbonneau, D. Kinet, Y. Hernandez, and D. Giannone, "All-normal dispersion, all-fibered PM laser mode-locked by SESAM," Opt. Express, vol. 20, pp. 11918-11923, May. 2012.

[6] M. M. Keshk, I. A. Ashry, M. H. Aly, and A. M. Okaz, "Analysis of different fiber Bragg gratings for use in a multi-wavelength Erbium doped fiber laser," in Proc. of IEEE Conf. on Radio Science, Cairo, 2007, pp. 1-13.

[7] I. Ashry, and H. M. H. Shalaby, "All-optical variable delay buffer for next generation optical networks," in Proc. of IEEE Conf. on Transparent Optical Network, Munich, 2010, pp. 1-3.

[8] I. Ashry, and H. M. H. Shalaby, "Tunable Fabry-Perot interferometer based on fiber Bragg gratings," in Proc. of IEEE Conf. on Telecommunications (ICT), Doha, 2010, pp. 543-537.

[9] P. Orr, and P. Niewczas, "High-speed, solid state, interferometric interrogator and multiplexer for fiber Bragg grating sensors," $J$. Lightwave Technol, vol. 29, pp. 3387-3392, Nov. 2011.

[10] A. F. Goncalves, L. A. Ferreira, F. M. M. Araujo, P. M. Mendes, and J. H. Correia, "A smart skin PVC foil based on FBG sensors for monitoring strain and temperature," IEEE Trans. On Industrial Elect., vol. 58, pp. 2728-2735, July. 2011.

[11] N. A. Mohammed,T. A. Ali, and M. H. Aly , "Evaluation and performance enhancement for accurate FBG temperature sensor measurement with different apodization profiles in single and quasidistributed DWDM systems," Opt. and lasers in Engineering, vol. 58, pp. 22-34, Jan. 2014.

[12] D. L. Aybatov, R. R. Kiyamova, O. G. Morozov, and E. V. Suhorukova, "Tunable Fabry-Perot interferometer based on fiber Bragg gratings," in Proc. of SPIE Conf. on Optical Technologies for Telecommunications, 2009.

[13] M. Ferchichi, M. Najar, and H. Rezig, "Design of temperature-strain tunable UDWDM, DWDM, WDM FBG filter for Passive Optical Network Access," ICTON, Marrakech, 2008, pp. 1-5. 